\title{
Analysis of the 3D Simulation Reconstruction Method for Road Surface Roughness
}

\author{
Lan-ying ZHAO* \\ College of mechanical and power engineering \\ Dalian Ocean University \\ Dalian, Shahekou District, China \\ e-mail: zhaolanying@edu.com.cn, www.dlou.edu.cn \\ Li-xiu CHEN \\ College of mechanical and power engineering \\ Dalian Ocean University \\ Dalian, Shahekou District, China

\section{Gang MU} \\ College of mechanical and power engineering \\ Dalian Ocean University \\ Dalian, Shahekou District, China
}

\author{
Jing-lun YANG \\ College of mechanical and power engineering \\ Dalian Ocean University \\ Dalian, Shahekou District, China
}

\author{
Jing-bo YU \\ College of mechanical and power engineering \\ Dalian Ocean University \\ Dalian, Shahekou District, China
}

\begin{abstract}
Establishing reasonable road roughness incentive model is the basis of the vehicle performance analysis and simulation experiment. It is very significant for the fields of vehicle engineering and road engineering to analyze on the road surface roughness. At present, Because of the lack of basic road data, the study of two-dimensional surface simulation has been relatively mature. Usually 3D simulation reconstruction was carried out based on two-dimensional surface raw data or two-dimensional surface model, and then to check models, and put them into virtual experiment or simulation application. Methods of the three-dimensional simulation reconstruction for Road roughness were summarized,and its characteristics and application were analyzed in this paper.
\end{abstract}

Keywords-Road surface roughness; $3 d$ simulation and reconstruction: Research status

\section{INTRODUCTION}

Road surface roughness is both one of the evaluation indexes of the road project quality at both domestic and international countries, and in the field of vehicle engineering road roughness is the main factor in the environment of vehicle operating and the main sources of incentive and vibration. Therefore accurate representation and modeling for road surface roughness is the foundation of the vehicle comfort, vehicle and system dynamics analysis and pavement assessment. In the research of road surface roughness simulation, many domestic and foreign experts and scholars have done a lot of research which has gone through from the frequency domain to the time domain, from linear to nonlinear, from smooth to non-stationary, from two-dimensional simulation to three-dimensional simulation process.

The frequency domain model (harmonic superposition model [1,2], trigonometric series method [3, 4], white noise model [5] and power spectral density conversion based model [6] is usually applied to vehicle dynamic response analysis. In the study of compliance, the corresponding vibration system input also uses the frequency domain road spectrum, the frequency domain model statistic method is mature, but only applies to the linear system. Time domain model (AR / ARMA model [7, 8]) can be used to calculate the response of the system to the time history by time-integration of the system dynamics equation, which is suitable for the analysis and simulation of nonlinear vehicle ride comfort dynamics. When driving with acceleration, deceleration, starting and braking, the road excitation will be a non-stationary random process, and Lei Yuliang [9] used the Winger spectral model to generate the non-stationary random road surface. Zhang Lijun and Zhang Tian-xia [10] has established a non-stationary universal time series model with input covariance, analyzing the instantaneous power spectrum Ma Junfu and Zhou Xiaojun [11] used the relationship between DHM (Davies-Harte Method) and road roughness of smooth and non-smooth FD (Fractionally Differenced) process to simulate the pavement roughness of smooth and non-stationary FD process. Wang Guolin et al [12] has established a non-stationary four-wheel correlation filtering white noise pavement random excitation time-domain model, combined with the single-point discrete time-domain model of vehicle co-simulation for vehicle ride comfort prediction, simulation and parameter system parameter optimization. Zhang Yonglin et al [13] carried out efficient numerical simulation of spatially correlated vehicle road.

The degree of simulation has been gradually expanded with the development of the computer simulation software and simulation level the needs of vehicle vibration and system dynamics in dept research. In this paper, the characteristics of three-dimensional simulation 
reconstruction method of road roughness are analyzed. The way of obtaining raw data of pavement, the three-dimensional construction method of pavement and the application of model verification method and model are summarized.

\section{THE SIMULATION OF RECONSTRUCTION OF THE 3D DIMENSIONAL ROAD SURFACE ROUGHNESS}

According to the present situation of research, the simulation reconstruction of $3 \mathrm{D}$ road roughness is summarized as shown in Fig.1. Firstly, the original data of pavement would be obtained, the 3D data was reconstructed based on the original data, and then the reconstructed model will be validated, using the virtual test field or three-dimensional simulation software.

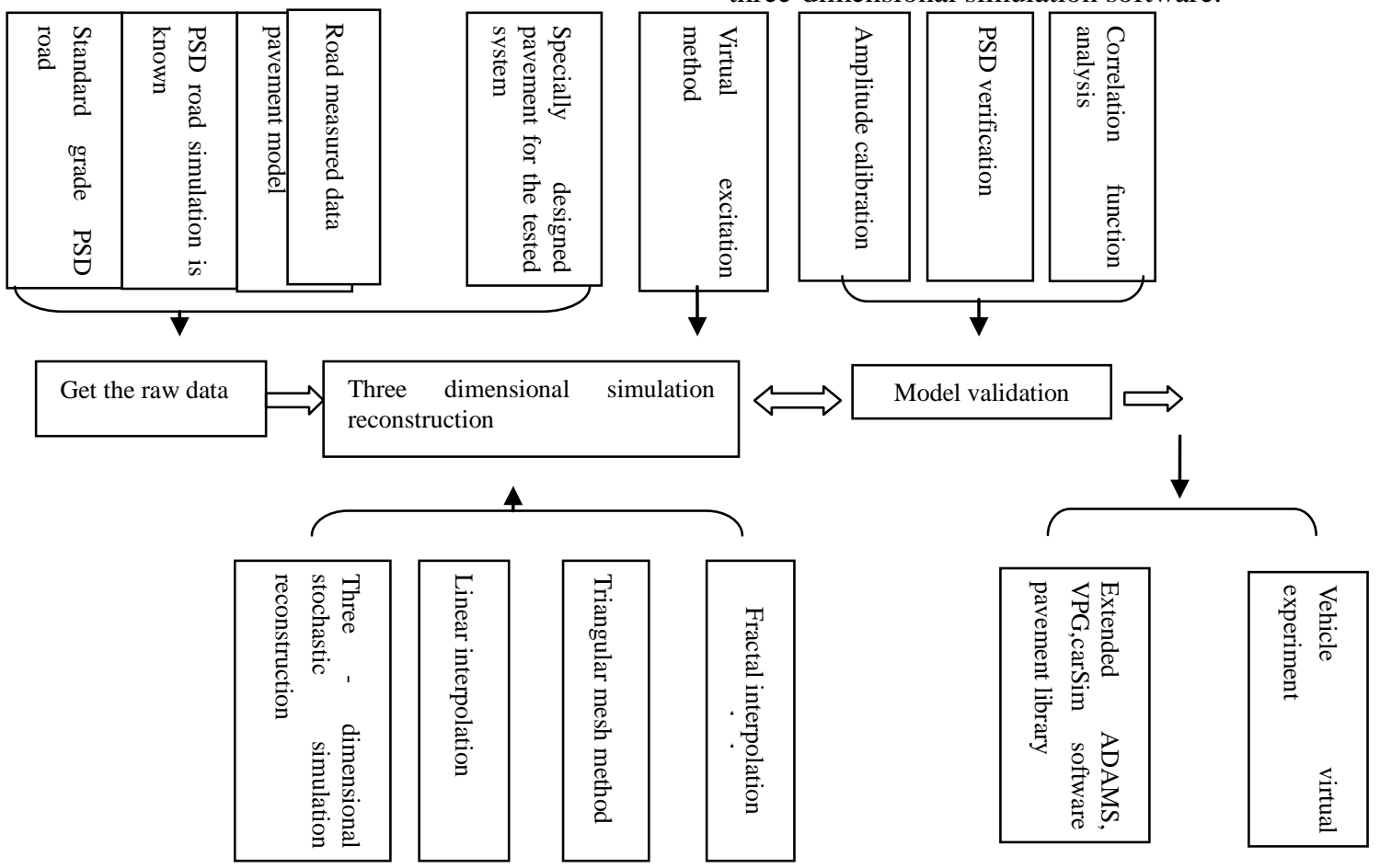

Figure 1. Process of 3D simulation reconstruction for road surface roughness

\section{ANALYSIS OF THREE - DIMENSIONAL ROAD SURFACE ROUGHNESS SIMULATION RECONSTRUCTION METHOD}

\section{A. Three - Dimensional Road Surface Roughness Simulation Based on Stochastic Excitation and Two - dimensional Pavement Model}

Zhang Yonglin et al. [14] has constructed a three-dimensional time series model based on the dual-independent time series model, which has realized the reconstruction of any stationary random road. The method assumes a wide stationary random field, zero mean, On the assumption that the pavement is isotropic, five road traces in different directions were used to characterize the pavement, and a linear stochastic pavement was obtained by linear interpolation., For the known pavement, it can take five different directions of the track, according to sampling theorem sampling, as the three-dimensional time series modeling method of the original data.. Peng Jia et al [15] based on single-point FFT road surface roughness time-domain model to establish a three-dimensional FFT random road surface input model to achieve a three-dimensional FFT random road surface MATLAB simulation, and import ADAMS software to build three-dimensional pavement simulation environment, vehicle prototype of the ride simulation. XueJinlu et al. [16] applied the harmonic superposition method to build a 3-D stochastic pavement model which is suitable for ADAMS software tracked vehicle module. The model can be used to simulate the vertical and lateral load of the vehicle, compared with the two-dimensional pavement, which has made a big improvement. For the three-dimensional random pavement, the pavement surface roughness cannot simulate the variation of the road roughness, and it has a great influence on the virtual test result. For the pavement with certain shape, the change of the number of the pavement will affect the pavement precision. Liu Dawei et al. [17] established a 
three-dimensional stochastic pavement mathematical model based on the basic principle of harmonic superposition method and triangular mesh method when carrying out road-friendly simulation analysis of road heavy-duty vehicles. The results of AR spectrum analysis and coherence analysis showed that the three - dimensional random pavement conforms to the actual situation of the road surface. Jiang Rongchao et al. [18] established a three-dimensional virtual road deceleration zone model by triangular mesh method, generated three-dimensional virtual road deceleration zone containing road surface information, and established a three-dimensional stochastic pavement mathematical model based on harmonic superposition method. The three-dimensional pavement isomorphism model provides the basis for the study of vehicle-road deceleration zone coupling using related application software.

\section{B. Three - Dimensional Pavement Reconstruction Based on Measured Pavement}

Due to the limited number of pavement models available in multi-body dynamics software, it is difficult to meet all the requirements of vehicle simulation. Chen Chao et al. [19] proposed a pavement file synthesis method based on actual collected rough-surface road roughness data. The method can be used to construct three-dimensional pavement model with special requirements in complex shape and dynamic simulation, which can meet the requirement of pavement model in practical work. However, the method of writing pavement files involves a large number of nodes and the construction of the unit is complex, it is necessary to write a pavement file generation program to read the road surface roughness data and generate the corresponding pavement files.

HouYongtang et al. [20] established the AR model in one-dimensional space coordinate system and the AR model in two-dimensional space coordinate system, reconstructed one-dimensional and two-dimensional standard road and measured road surface. With 3D virtual road, the system can directly generate the pavement three kinds of files named VPG, CarSim and ADAMS, and provide a rich 3D road surface input file for the vehicle dynamic simulation analysis software. Finally, the software of 3D virtual road reconstruction software is developed.

\section{Establishment of Road Surface Roughness Model by Virtual Excitation}

The three-dimensional stochastic Road Surface Roughness model constructed by the discrete-sampling road simulation method [21] ("PEM" method) of virtual excitation method is as follows:

$$
q(x, y)=\sum_{k=0}^{N_{\mathrm{r}}-1} \sqrt{G_{q q}(f=k \Delta f)} e^{i\left(\frac{2 \pi k x}{X}\right)}
$$

In which, $\mathrm{x}$ is the longitudinal coordinate, $\mathrm{y}$ is the horizontal coordinate, is the total sampling points, $\mathrm{k}$ is the non-negative integer, is the bilateral power spectrum, $\mathrm{f}$ is the frequency, is the sampling frequency, $X$ is the road longitudinal total length.

The virtual excitation method can quickly and effectively analyze such vibration, and overcome the shortcomings of poor accuracy, difficulty and low efficiency in the random vibration analysis. The virtual excitation method can quickly and effectively analyze such vibration, and overcome the shortcomings of poor accuracy, difficulty and low efficiency in the random vibration analysis. Li et al. [22] used virtual excitation method to construct the virtual pavement excitation, and established the power spectral density formula to obtain the system's vibration response. Lin Min et al [23] based on the existing virtual 2D pavement spectrum and the improved virtual exciting method from the frequency spectrum of space pavement, expand it to the pavement spectrum in three-dimensional space, give the simulation of random road spectrum method.

\section{Three - Dimensional Road Surface Roughness \\ Reconstruction Method Based on Fractal Feature}

With the development of the new theory and method, it has provided a new way for the establishment of pavement input model, the comprehensive evaluation of pavement performance and the establishment of pavement database system. With the development of the new theory and method, which has provided a new way for the establishment of road surface input model, the comprehensive evaluation of pavement performance and the establishment of pavement database system. Fractal theory is a new branch of mathematics theory, it is widely used in signal description, signal processing and many other fields. And its combination with the surface roughness of the study has become an important cross-branch of scientific research. Many studies have shown that the measured road surface roughness has self-similarity and self-affine characteristics, and its fractal dimension reflects the natural characteristics of road roughness. Combining the traditional characterization parameters with fractal dimension can be more realistic to characterize road surface unevenness degree of the characteristics of road surface roughness [24-26]. Fractal model also plays an important role in the study of road roughness simulation, WM function, fractal Brown model, fractal interpolation function and other two-dimensional fractal model has been used in the simulation of the measured road and vehicle dynamic load simulation analysis [24].The better accuracy and the applicability of vehicle ride comfort analysis. The application of pavement roughness fractal model can also make the related research field get the road surface excitation closer to the real road surface. The two-dimensional pavement fractal model has been applied in the research of vehicle multi-body dynamics, Le Gala[27], D Besdo [28] established a rough self-affine pavement for analysis of friction characteristics. Due to the complexity of the model parameters, it is not suitable for the research of vehicle pavement systems. Ma Bin [29] proposed a new method combining tire fractal characteristics and tire characteristics the fractal dimension of pavement roughness is one of the factors that influence the effect of pavement on the rubber-induced friction. 3D simulation of road roughness 
based on fractal theory has also been carried out initially. $\mathrm{Li}$ Xiaoqin [30] applied the method of random midpoint displacement and triangulation to establish a three-dimensional theoretical model of the pavement surface of traffic vehicles. (RMD) was used to generate three-dimensional pavement based on the average fractal dimension $\mathrm{D}=1.59$ of the simulated standard grade pavement obtained by the square root method. Triangular meshes were used as the structural framework to generate the data. Dimensional pavement model, however, in the model, the same fractal dimension of the curve is adopted in the model, so the method of constructing it needs to be further improved, and the method of constructing the model needs to be further improved. Its practical value needs to verify. In the current mainstream multi-body dynamics software, 3D pavement is generally discredited into finite triangular elements. In theory, any shape of road can be expressed by a finite number of triangular element combinations, so the study of triangular element generation and the splicing method is the key to study the pavement model construction in multi-body dynamics software. 3D Fractal Brown model, 3D fractal interpolation method [31, 32], subdivision random displacement method [33] and so on in terrain analysis will have further application in road surface roughness 3D reconstruction.

\section{SUMMARY AND PROSPECT}

- As the research and application of two-dimensional pavement model is more mature, the method of three-dimensional pavement model based on stochastic pavement model and two-dimensional pavement model is easy to be realized, which can meet the needs of the initial research, but cannot reflect the actual three-dimensional pavement.

- Three-dimensional reconstruction method based on measured road surface has just started. This method can obtain three-dimensional pavement which retains certain measured pavement characteristics. The pavement files are enriched by the pavement database of vehicle simulation and analysis software, and the research is mainly aimed at Corresponding measures, has not yet been measured on the road sampling interval, the sampling length of uniform, while the comparison of the sampling data of the test equipment yet to be studied.

- Three-dimensional road surface roughness simulation technology based on new theory and new method has been gradually developed. Virtual excitation method and fractal theory have been applied in pavement roughness simulation. The three-dimensional simulation method of pavement roughness based on fractal features, Dimensional fractal characteristics of road surface roughness, the purpose of which is to establish a three-dimensional pavement model which combines the traditional characteristics of road roughness and fractal characteristics. This research needs further study; three-dimensional fractal reconstruction algorithm has broad application prospects in three-dimensional pavement roughness simulation reconstruction.

In short, the simulation of real three-dimensional road roughness is paid more and more attention. The three-dimensional representation of road surface, the reconstruction of simulation and the establishment of open three-dimensional virtual road database will be further studied and applied.

\section{ACKNOWLEDGMENT}

This work is supported by the general project of Liaoning province department of education (L2015088); Fund of Liaoning province (201602108)

\section{REFERENCES}

[1] Zhang Yonglin, Time domain model of road irregularities simulated usingthe harmony superposition method [J], Transactions of the Chinese Society of Agricultural Engineering, 2003, Vol.19 No.6, pp. $32-35$

[2] Chang Zhiquan, Luo Hong, CHU Zhi-gang, DENG Zhao-xian, Building Model of Road Roughness [J], Journal of Chongqing University (Natural Science Edition), 2004, Vol.27No.12, pp. 5-8

[3] Gao Nong, Liu Mingsi, A Method of Computer Imitation for Automobile Random Vibration at not level Rood Surface [J], Natural Sciences Journal of Harbin Normal university, 1997, Vol.13 No.5, pp. 101 104

[4] Zhang Yonglin, Zhong Yifang, Time Domain Model of Road Undulation Excitation to Vehicles [J], Transactions of the Chinese Society for Agricultural Machinery, 2004, Vol.35 No.2,pp. 9 12

[5] Zhang Guosheng,Fang Zongde,Chen Shanzhi, Song Yunqiang, White Noise Simulation for Road Roughness Based on Power Function [J], Automotive Engineering, 2008, Vol.30No.1, pp. 44-47

[6] Liu Xiandong, Deng Zhidang, Gaofeng, Study of simulation of road roughness based on inversetransform [J], China Journal of Highway and Transport, 2005,Vol. 18No.1,pp. 122 126.

[7] Wang Baiyi,Wu Weisen, A New method for Simulating Road roughness by Using $\mathrm{AR}(\mathrm{P}, \mathrm{O})$ Series[J],Journal of Wuhan Institute of Technology, 1987, No.2, pp. 60-68

[8] Tang Guangwu, He Xuefeng, Yan Yongfu, Mathematical model of road roughness in time domain and its simulation [J],China Journal of Highway and Transport, 2000, Vol.13No.1, pp. 114-116

[9] Lei Liangyu, Zhang Weiping, Wang Jiangtao, Simulation and Analyses on Nonstationary Random Road [J], Chiness Journal of Sensors and Actuators, 2007, Vol.20No.4, pp. 877-880

[10] Zhang Lijun, Zhang Tianxia, Study on General Model of Random Inputs of the Vehicle with Four Wheels Correlated in Time Domain [J], Transactions of the Chinese Society for Agricultural Machinery, 2005, Vol.36 No.12, pp. 29-32.

[11] Ma Junfu, Zhou Xiaojun, Xu Zhinong, A Study on the Simulation and Recognition ofRoad Roughness [J], Machine tool and Hydraulics, 2008, Vol.36 No.1, pp. 143-145

[12] Wang Guolin, HuJiao, Qian Jinge, Wang Yiqi, Road disturbances of non-stationary excitation time domain simulation and wavelet analysis [J], Journal of Vibration and Shock, 2010, Vol.29No.7,pp. 28-32

[13] Zhang Yonglin, Hu Zhigang, Chen Liping, A New Approach to Numerical Simulation of the Vehicle Road Process with Temporal-spatial Correlation [J], Transactions of the Chinese Society for Agricultural Machinery 2005, Vol.36 No.9, pp. 13-15

[14] Zhang Yonglin,Yi Qiwei,Ding Shiyun, Wang Wangping, Research on Time Series Modeling Method of 3D Stochastic Road [J], Journal of Wuhan University of Technology, 2007, Vol.29No.8, pp. 92-95

[15] Peng Jia, He Jie, Cong Ying, Li Xuhong, Chen Yikai, Modeling and Simulation of General 3D Virtual Stochastic Road Model [J], 
Transactions of the Chinese Society for Agricultural Machinery, 2009, Vol.40 No.3, pp. 1-4

[16] Xue Jinlu, Wang Hongyan, Chi Baoshan, Modeling Research on Road Used for Virtual Experiment of Tracked Vehicle [J], Automotive Engineering, 2012, Vol.34 No.7, pp. 647-651

[17] Liu Dawei Jiang Rongchao Chen Huanming Yan Tianyi, Simulation of Road-friendliness of Heavy Vehicle on 3-D Random Road [J], Transactions of the Chinese Society for Agricultural Machinery, 2012, Vol.43No.12, pp. 16-22

[18] Jiang Rongchao, Chen Huanming, Liu Dawei, Wang Song, Reconstruction and Implementation of 3D Virtual Road Speed Control Humps for Vehicle [J], Journal of Qingdao University (Engineering \& Technology Edition), 2012, Vol.27 No.1, pp. 32-36

[19] Chen Chao, Wei Laisheng, Zhao Taoshuo, Study on the Building Approach of the Road Modelin Multi-body Dynamics Simulation [J], Vehicle \& Power Technology, 2012, No.1, pp. 41-44

[20] Hou Yongtao, Wang Guolin, Liu Jian, Shao Changzheng, Design and Implementation of the 3D Virtual RoadReconstruction System for Vehicle [J], Journal of Mechanical Engineering, 2011, Vol.47 No.8, pp.126-132

[21] Zhao Yan,Zhang Yahui, Lin Jiahao, Summary on the Pseudo Excitation Method for Vehicle Random Vibration PSD Analysis[J], Applied Mathematics and Mechanics, 2013,Vol.34 No.2, pp.: 107-117

[22] Li Jie,Qin Yuying, Zhao Qi, Zhang Wei, A Kind of new Method Used for Analyzing random vibration of vehicles [J], Journal of Machine Design, 2009, Vol.26 No.4, pp. 14-17

[23] Lin Min, Zhang Xiangwei, Cheng Siyuan, Simulation of Three-dimensional road based on pseudo excitation method [J], Machinery Design \& Manufacture, 2012, No.2, pp. 84-86

[24] Lu Zhixiong, Zhao Lan-ying, Hou Zhanfeng, Fractal Behavior of Road Profile Roughness [J], Journal of Jiangsu University (Natural Science Edition), 2008, Vol.29 No.2, pp. 111-114
[25] Hou Zhanfeng Lu Zhixiong Zhao Lanying, Fractal Behavior of Tillage Soil Surface Roughness [J], Transactions of the Chinese Society for Agricultural Machinery, 2007, Vol.38 No.4, pp. 50-53

[26] LU Zhixiong, ZHAO Lanying. On fractal behavior of road surface roughness. International conference on Transportation Engineering [C]. 2007, Vol4, pp. 3464-3468.

[27] Le Gala, L.Guyb, G. Orangeb. Modelling of sliding friction for carbom black and silica filled elastomers on road tracks [J]. Wear, 2008, Vol.264 No.7, pp. 606-615

[28] D. Besdo. Modelling of Dry and wet friction of silica filled elastomers on self-affine road surfaces [J]. Elastomere Friction, 2010,pp. 1-26

[29] Ma Bin, Xu Hongguo, Liu Hongfei, Effects of Road Surface Fractal and Rubber Characteristics on Tire Sliding Friction Factor [J], Journal of Jilin University (Engineering and Technology Edition), 2013, Vol.43 No.2, pp. 317-322

[30] Li Xiaoqin, Lu Zhixiong, Xi Xinxin, Zhao Miaomiao, 3D reconstruction of transportation vehicles travecting pavement based on fractal characteristics $[\mathrm{J}]$, Transactions of the Chinese Society of Agricultural Engineering, 2012, Vol.28 No.2, pp. 61-65

[31] Liang Jun, Wang Qi, Liu Kunliang, Lu Quanhui, 3D Terrain Simulation Based on the Method ofRandom Mid-point Displacement [J], Computer Simulation, 2005, Vol.22 No.1, pp. 213-240

[32] Y.M.Huang,Ching-Ju Chen. 3D Fractal reconstruction of terrain profile data based on digital elevation model [J].Chaos Solitons and Fractals, 2009, pp. 1741-1749

[33] Zhang Runhua, Liu Shuqun, Zhao Fuqing, Terrain generation based on the method of random subdivision displacement [J], Journal of Huazhong Normal University (Natural Sciences), 2012, Vol.46 No.5, pp. 533-536 